Review Article

J Exp Clin Med

2021; 38(S2): 92-97

doi: $10.52142 /$ omujecm.38.si.dent.3

\title{
Clinical outcomes and complications of CAD-CAM fabricated complete dentures: An update and review
}

\author{
Emir YÜZBAŞIOĞLU ${ }^{1,2^{*}}$, Yeşim ÖLÇER US ${ }^{1}$ ๑, Gökhan ÖZDEMİR ${ }^{1}$, Berkman ALBAYRAK ${ }^{1}$
}

${ }^{1}$ Department of Prosthodontics, School of Dental Medicine, Bahçeşehir University, Istanbul, Turkey

${ }^{2}$ Department of Dentistry, School of Dental Medicine and Health Sciences, BAU International University, Batumi, Georgia

\begin{abstract}
\begin{tabular}{ccccc}
\hline Received: 03.05 .2020 & $\bullet$ & Accepted/Published Online: 04.12.2021 & $\bullet$ & Final Version: 19.05 .2021 \\
\hline
\end{tabular}
Abstract

For decades, conventional complete dentures (CD) have been a promising treatment for edentulous patients. The introduction of digital technology in $\mathrm{CD}$ fabrication streamlines and simplifies the treatment process and offers new and specific applications for the completely edentulous patients. Computer-aided design/computer-assisted manufactured (CAD/CAM) CD protocols can improve efficiency and offer specific applications in specific situations to improve patient care, satisfaction, and convenience. The aim of this review is to assess and evaluate the clinical outcomes and complication of $\mathrm{CAD} / \mathrm{CAM}$ fabricated $\mathrm{CD}$ systems and to provide information about currently available systems for dental practitioners.
\end{abstract}

Keywords: CAD-CAM, complete denture, clinical outcome, complication

\section{Introduction}

Edentulism, or the absence of teeth, contributes to disability, impairment, and handicap that significantly affect the general health and overall quality of life of an individual (Viola et al., 2013). Complete denture (CD) is the most common prosthodontic therapy in edentulous patients with anatomical, psychological or financial constraints contradicting implant therapy; many essential therapeutic variables have not yet been scientifically validated (Abduo, 2013). The use of complete dentures for rehabilitation of edentulous patients has acceptable outcomes for most patients (Muller, 2014).

For decades, conventional CD's have been a promising treatment for edentulous patients. In addition to having advantages such as verifying each stage and customizing teeth positions during the construction of complete dentures before delivery, the existence of various disadvantages should also be taken into accounts such as multiple appointments, uncertain laboratory costs and time (Bidra et al., 2013).

A numerical control device, PRONTO, was introduced in the late 1950s which indicates beginning of computer-assisted design/computer-assisted manufacturing (CAD/CAM) era. The very first CAM software program to be developed by Dr. Patrick J. Hanratty, considered as the father of CAD/CAM (www.cadazz.com, 2020). CAD technology is a widely term which refers the use of computers to aid in the creation, analysis, and manufacture of design (Dankwort et al., 2004). The major components of CAD/CAM technology are listed below (Alghazzawi, 2016):
1. Data acquisition unit for acquiring the data of anatomical or dental structures by using intraoral scanners or indirectly digitalizing of a stone model

2. Softwares; used in designing virtual restorations for manufacturing.

3. Computerized manufacturing device; is used for manufacturing the restorations.

$\mathrm{CAD} / \mathrm{CAM}$ technology based on two main system are subtractive manufacturing and additive manufacturing. The subtractive manufacturing is a process by which $3 \mathrm{D}$ objects are created by machining to cut material away from a solid block material achieve the desired geometry as CNC (computerized numerical control) machine. The subtractive manufacturing is widely used in dentistry, especially in prosthodontics. However, limited number of authors have reported the use of CAD/CAM technology for complete dentures (Maeda et al., 1994; Kawahata et al., 1997; Busch et al., 2006; Sun et al., 2009; Zhang et al., 2011; Kanazawa et al., 2011; Goodacre et al., 2012; Inokoshi et al., 2012).

In prosthodontics, the subtractive manufacturing is widely used. However, limited number of authors have reported the use of CAD/CAM technology for CD's (Maeda et al., 1994; Kawahata et al., 1997; Busch et al., 2006; Sun et al., 2009; Zhang et al., 2011; Kanazawa et al., 2011; Goodacre et al., 2012; Inokoshi et al., 2012). This is possibly occasioned by because of complicated manufacturing of CDs. Because many factors have an important effect on them as multiple steps of 
recording, transferring, analyzing, and afterward replacing of artificial substitutes for gingiva and teeth, all of which must be in harmony with patient's mouth and face. All these determinants need the dentist's technical and artistic skill, which is less handy adapted to CAD/CAM than intracoronal or tooth-supported restorations.

The very first scientific article on the use of a CAD/CAM systems for CD production was published by Maeda et al., (1994), and these first CAD/CAM fabricated CDs were made by additive rapid prototyping (RP) technology, from photopolymerized acrylate material using a 3-D laser lithographic (LL) machine (Maeda et al., 1994). Since then, because of the complexity of the procedures for fabricating $\mathrm{CDs}$, it took almost 20 years for the emergence of the first commercially available denture systems. Katadiyil et al. (2013) presented the procedures for the production of $\mathrm{CDs}$ for the first two commercial CAD/CAM systems (AvaDent \& Dentca) which are available in the market. The fabricating process of digital CDs consists of scanning the definitive impressions or casts and a maxillomandibular record, designing the denture base and arranging the artificial teeth by using a software program, and then manufacturing the denture by using either an additive (3D-printing) or subtractive (computerized numerical control milling) technique (Kattadiyil et al., 2013; Bilgin et al., 2015; Alghazzawi, 2016; Wimmer et al., 2016). The digitalized process can not only reduce the clinical appointments and chair time but also improve visualization and detection of the edentulous arch morphology and also decrease the polymerization shrinkage of the polymethyl methacrylate (PMMA) (Steinmassl et al., 2017). In conventional fabrication techniques, factors such as detailing the accuracy of $\mathrm{CAD} / \mathrm{CAM}$ fabricated $\mathrm{CDs}$, and reliable in vivo studies are scarce and have used a subjective rating scale to assess the retention and fit of dentures, making blinded evaluation impossible (Kattadiyil et al., 2015; Schwindling and Stober, 2016).

There are several advantages of $\mathrm{CAD} / \mathrm{CAM}$ fabricated CDs over conventional CD's.

1. Reduced travel expense for the patient due to fewer appointments (Bidra et al., 2013).

2. The adaptation of $\mathrm{CAD} / \mathrm{CAM}$ fabricated $\mathrm{CDs}$ to underlying tissues are good (Bidra et al., 2013).

3. Reduced micro-porosity of prepolymerized PMMA blocks (Steinmassl et al., 2017).

4. The decrease in accumulation of Candida albicans compared to conventional processing technique (Bidra et al., 2013).

5. The storage of digital files which allows fabrication of new prosthesis when dentures are lost or damaged (Infante et al., 2014).

There are some limitations and disadvantages of the current commercial manufacturing systems that need to be considered.
6. It is challenging to assess the vertical dimension, maxillo-mandibular relation, lip support and maxillar incisal edge position.

7. It is almost impossible to establish the mandibular occlusal plane.

8. Patients have minimal opportunity to participate in the procedure.

9. High investment costs and laboratory costs compared to conventional techniques (Bidra et al., 2013).

Recently there are six commercial workflows for digital complete dentures on the market: AvaDent (Global Dental Science), Ceramill Full Denture System (Amann Girrbach $\mathrm{AG}$ ), Baltic Denture System (Merz Dental $\mathrm{GmbH}$ ), DENTCA/Whole You (DENTCA, Inc.; Whole You, Inc), Wieland Digital Denture (Ivoclar Vivadent, Inc). And Vita Vionic (VITA Zahnfabrik).

\section{Wieland digital denture (Wieland dental + technikivoclar vivadent)}

Wieland Digital Denture protocol is able to finalize a digital denture delivery in four patient visits. Conventional impression trays are used to perform alginate impressions in the first visit. Also jaw relation and vertical records are determined by a centric tray and occlusal plane is determined using a special provisional transfer arch (UTS CAD). Impression trays with the jaw record and occlusal plane information are milled to be used for functional impression in second visit. If needed jaw record and occlusal plane adjustments can be done regarding the compensation values of UTS CAD transfer arch. Next step of second visit is gothic arch registration via functional impression and gnathometer CAD. Third visit is an optional try-in session, if needed dentures milled from PMMA are prone to minor adjustments and delivery to patient possible at third or fourth visit.

\subsection{AvaDent digital dentures (Global dental science)}

Global Dental Science offers complete digital dentures delivery in three visits. The workflow ensures to finalize the denture in two sessions if try-in session is skipped. Vertical dimension is determined by measuring two points on nose and chin and process is followed by functional impression taking by individual thermoplastic trays. Centric relation is recorded by gothic arc as well as vertical dimension is reproduced taken into account the lip support and occlusal plane with anatomical measuring device (AMD) or wax rim. At second appointment it is possible to perform a PMMA try-in which is optional. If the clinician doesn't prefer a try-in session, visualizing the final denture by fixing cellophane resembling teeth to the AMD is possible.

\subsection{Whole you nexteeth (Whole You; Formerly DENTCA)} The workflow is similar to the previous ones. At first session vertical height is evaluated and recorded with the help of reference marks on upper and lower lips. Special trays are used for functional impression. Vertical occlusal dimension is then 
needing to be reproduced by using the integrating stylus in the lower tray, placed for final adjustments in patient. Gothic arch recording is performed on a plane adapted in the upper tray. Lip support and occlusal plane information is provided automatically from impression. Clinician may add other anatomical reference information like incisor edge length. For esthetic and functional try-in 3D printed acrylic try-in dentures can be used. The system then produces a milled denture with bonded teeth.

\subsection{Baltic denture system (Merz Dental)}

Baltic Denture System uses bite rims with relined occlusal arches to obtain the ideal 3D position. These occlusal arches have prefabricated different size tooth set-ups that can be adjusted. During first session, the vertical occlusal dimension is obtained and functional impressions are performed. By supporting the BD Upper Key (a bite rim resembling the final maxillary dental arch) with silicone impression material or thermoplastic impression compound, the occlusal plane, incisor length, and lip support are determined. When the maxillary dental arch has been adjusted to the ideal position, the BD Lower Key (a bite rim resembling the final mandibular dental arch) is interlocked with the BD Upper Key by click mechanism and, again, supported with silicone impression material to reach the previously determined vertical dimension and the centric maxillo-mandibular relation. Since the BD Keys resemble the final dental arches, the adjusted keys also serve as try-in dentures so that the final dentures can be placed at the next appointment.

\subsection{Ceramill full denture system}

The digital workflow starts with the digitization of maxillary and mandibular casts and virtual positioning of teeth and waxing of polished surfaces (Wimmer et al.,2016). Bases of dentures are milled from wax and teeth are placed in the sockets on base. Any anatomical features can be designed and mills on wax. Afterwards dentures are produced conventionally.

\subsection{Vita vionic (Vita zahnfabrik)}

In VITA VIONIC the digital design and production can be facilitated by non-system-inherent scanners, software, and milling machines. The conventional production protocol is defined in five steps and can also be finalized with reduced sessions only in three sessions. The simplified workflow can be summarized as anatomical and functional impression, vertical dimension and centric relation and denture delivery. Try-in dentures can be milled from Vita wax.

\section{Clinical outcomes of CAD-CAM complete dentures}

Several researches investigated clinical outcomes of CAD/CAM fabricated CDs based on patient centered outcomes and time efficiency. Kattadiyil et al. (2015) stated dramatically increased retention when comparing milled maxillary CAD/CAM fabricated CDs with the conventional $\mathrm{CD}$ manufactured for the same patient. In a pilot cohort study of satisfaction-stability, retention and adaptation relationship were reported that $50 \%$ of patients did not rate as either good or excellent Bidra et al. (2016). Schwindling and Stober (2016) stated that milled maxillary CAD/CAM fabricated CDs were slightly more retentive than maxillary injection molding CDs. Saponaro et al. (2016) reported that in 48 (2.08\%) patients; one had impaired phonetics and $3(6.25 \%)$ stated poor aesthetic results with their CAD/CAM fabricated CDs. Kattadiyil et al. (2015) and Schwindling and Stober (2016) found no remarkable differences between milled CAD/CAM fabricated CDs and other kind of CDs in view of phonetics and esthetics. Bidra et al (2015) stated great feedbacks for esthetics and phonetics with CAD/CAM fabricated CDs.

Bidra et al (2016) reported 15\% (3/20) of patients was dissatisfied of CAD/CAM fabricated CDs was (two of hysterical, and one of exacting had not satisfaction). Saponaro et al. (2016) assessed requirements of post-insertion adjustment visit in their study, 6/48 (12.5\%) patients did not require any post-insertion adjustment visit, 16/48 (33.33\%) patients needed merely 1 post-insertion visit, 14/48 (29.16\%) patients needed 2 visits, and 12/48 (25.00\%) needed 3 or more visits. In regard of these data, post placement adjustment visits resulted in a mean number of 2.08 (Saponaro et al., 2016). Bidra et al. (2016) stated in their study that a mean of 3.3 denture adjustments were needed more than 1 year for the 14 patients, of which $1 / 14(7.14 \%)$ patients needed a notable number of visits for denture adjustments. In their study, some researchers reported that $14.28 \%$ of patients requested additional $\left(3^{\text {rd }}\right)$ appointment, while others reported an average of 2.39 appointments required for the nominal 2-appointment protocol (Bidra et al., 2016; Saponaro et al., 2016). Similarly, Schwindling and Stober (2016) reported an average of 5.4 appointments for the 4-appointment protocol.

Kattadiyil et al. (2015) achieved 80\% satisfaction in their study with 15 patients, and similarly Bidra et al. (2016) achieved $79 \%$ satisfaction in their study with 14 patients. Other disadvantages mentioned in the studies related to CAD/CAM fabricated CDs have been reported as the need for immediate reline, disparities with OVD, problems with aesthetics, dental layouts and phonetics. (Kattadiyil et al., 2015; Saponaro et al., 2016; Bidra et al., 2016; Schwindling and Stober, 2016).

The current literature has shown the benefits that CAD/CAM fabricated CDs offer: fewer patient visits, digitally saved files for easier remakes, improved denture base adaptation, and improved retention, to name a few (Goodacre et al., 2012; Baba, 2016; Bidra et al., 2013, 2016). One of the most important advantages observed about CAD/CAM fabricated CDs is that fewer appointments (2) are required compared to the traditional method (5 appointments). Researchers reported an average of 2.39 appointments for patients treated by dentists during their postgraduate and predoctoral training. (Saponaro et al., 2016).

In a study related to the production time of $\mathrm{CAD} / \mathrm{CAM}$ fabricated CDs, the researchers stated that they saved a 
significant amount of time (approximately 205 minutes) compared to CDs prepared by the traditional method. It was reported that students were able to easily produce CAD/CAM fabricated CDs during the 2-appointment period under the guidance of the faculty during their predoctoral education (Kattadiyil et al., 2015).

In another study, it was stated that the time spent in the clinic in the production of CAD/CAM fabricated CDs decreased, but contrary to this advantage, the time spent in communication with the laboratory increased in order to achieve a successful result (Bidra et al., 2016).

Schwindling and Stober (2016) concluded that dentists who do not have sufficient experience with $\mathrm{CAD} / \mathrm{CAM}$ fabricated CDs use may need additional appointments in a study on a 4appointment CAD-CAM system that includes a trial placement appointment. In addition, in their pilot study, they reported that an average of 5.4 appointments were required to complete the production period.

In many studies, advanced initial fit and retention, attributable to polymerization shrinkage deficiency, has been identified as an important advantage of milled denture bases. (Kattadiyil et al., 2015; Saponaro et al., 2016; Bidra et al., 2016; Schwindling and Stober, 2016; Goodacre et al., 2016).

Fernandez et al. (2016) stated that the archiving of $\mathrm{CAD} / \mathrm{CAM}$ fabricated $\mathrm{CDs}$ is an important advantage compared to traditional CDs. The electronic archiving of all clinical data from the patient, together with the design of the manufactured prostheses, which enables making spare or new prostheses, in case of breaking or losing them, without clinical appointments. Bilgin et al. (2016), in their research, stated that microbial colonization should reduce with milled denture bases.

Similarly, milled denture bases have shown significantly higher short-term retention, (Kattadiyil et al., 2015) but longterm data were available in only 1 study of 1 -year duration (Bidra et al., 2016).

Al-Helal et al. (2016) reported that there was considerable improvement in retention to the CAD/CAM fabricated CDs compared with the traditional heat-polymerized CD's after 24 $\mathrm{h}$ storage in water before the testing appointment. The clinical outcomes demonstrated a considerable improvement in retention for the $\mathrm{CAD} / \mathrm{CAM}$ fabricated $\mathrm{CD}$ s compared with the traditional group. The authors suggested that feasible explanation for this might be the raised intensity of prepolymerized acrylic resin block, which offers higher dimensional stability and is not surely affected by hydration. He also noted that the arch form and type had no influence on the retention of either type of denture base.

Goodacre et al. (2016) compared the denture base adaptation of $\mathrm{CAD} / \mathrm{CAM}$ and conventional (pack and press, fluid resin, or injection) CD's an in vitro study and they concluded that CAD/CAM technology procreated the best accurate and reproducible dentures of the 4 tested techniques.

Al-Rumaih (2016) compared the retention of conventional and digitally milled CD's. He concluded that denture adhesive effectively compensates for potential differences in retention between conventionally and digitally designed denture bases.

Three common types of complication have been reported by Saponaro et al. (2016) lack of denture retention, incorrect centric relation (CR) and inaccurate vertical occlusal (VDO) dimension. The authors indicated that these complications could have been due to the overall quality of the impressions and the operator experience.

Srinivasan et al. (2017) compared the trueness of the intaglio surface CD's produced by different techniques (injection molding, flask-pack-press and $\mathrm{CAD} / \mathrm{CAM}$ ). they reported all techniques seems to remain within a clinically acceptable range CAD/CAM group showed the highest compression values (with the exception of the tuberosities), especially in the vestibular flange area. The vestibular flange (tighter inner seal) compression may be linked to improved retention.

Accordingly, the $\mathrm{CAD} / \mathrm{CAM}$ technique would be considered the best processing technique in comparison with traditional techniques. The results of Goodacre et al. (2018) for denture tooth movement demonstrated that techniques requiring compression during processing (pack-and-press, injection) showed increased positive occlusal tooth movement compared with techniques not involving compression (fluid resin, CAD-CAM bonded, CAD-CAM monolithic), meaning that it would cause an increase in the patient's OVD.

\subsection{Complications of CAD-CAM complete dentures}

Several researches investigated complications of CAD/CAM fabricated CDs such as lack of retention, necessity to relines, occlusal vertical dimension and centric relation errors, occlusion and tooth arrangement errors, esthetic and phonetic problems, tooth wear, additional visits to protocol, postinsertion adjustments, overall patient dissatisfaction and remake needed.

Saponaro et al. (2016) in a retrospective study, they stated that $16.66 \%$ of patients had inadequate prosthetic retention at the delivery appointment of the prosthesis. Bidra et al. (2016) in a prospective study, $50 \%$ of the patients stated that they did not feel sufficient prosthetic retention at delivery, and that 1 patient experienced significant retention loss after 1 year of follow-up. While Schwindling and Stober (2016) reported that $40 \%$ of their patients had prosthetic retention problems, in contrast, Kattadiyil et al. (2015) reported no problems with CAD/CAM fabricated CDs retention in a study with 15 patients.

Saponaro et al. (2016) found that 4 of the 48 participants had incompatibility in their occlusal vertical dimension. Besides, Schwindling and Stober (2016) stated that 2 of the 5 patients had an inadequate vertical dimension. Kattadiyil et al. 
(2015), found that wrong centric relationship record had been taken from one of the 15 participants. Likewise, the centric relationships of 3 of the 48 participants involved in the study of Saporano et al. (2016) were wrong. Bidra et al. (2016) noted that a higher proportion of centric relationship errors were revealed by $14.28 \%$ and extra sessions were conducted to confirm.

In their study, Schwindling and Stober (2016) attempted to establish bilateral balanced occlusion in the participants, but the need for intraoral adjustment was revealed in each participant. Although a rehearsal session was conducted to obtain a suitable occlusion, at least a single tooth for 2 of the 5 participants required to be corrected.

Saponaro et al. (2016) achieved inadequate aesthetic results in 3 of the 48 participants. Schwindling and Stober (2016) needed extra arrangements (no parallels of occlusal plane with Camper plane and interpupillar line, narrowed buccal corridors, midline deviation, and excessive support of lips) to improve esthetics in all 5 participants. Kattadiyil et al. (2015) however, reported that there is no significant difference between conventional and digital complete dentures in terms of esthetics.

Saporano et al. (2016) reported that they had regulated phonetics of one of the 48 participants $(2.08 \%)$ by applying heat-processed palatal reline due to the lack of palatal contour. Schwindling and Stober (2016) stated that one of the 5 participants had difficulty with pronouncing the letter "S" at first, but the situation improved without any alteration afterwards. A significant amount of wear on acrylic teeth of 3 of 14 participants was observed as a result of 1-year observation in the study conducted by Bidra et al. (2016).

Saponaro et al. (2016) stated that 17 of the 48 participants needed more than 2 sessions for CAD/CAM fabricated CDs; the 2-visit protocol was able to perform with an average of 2.39 visits. For 2 participants' rehearsal session was deemed necessary. Schwindling and Stober (2016) were able to complete their 4-session protocols with an average of 5.4 sessions. Saponaro et al. (2016) reported that 62.5\% (30 participants) needed a postinsertion adjustment for 1 or 2 sessions and 29.16\% (14 participants) needed for 3 or more sessions of 48 patients. On the other hand, Bidra et al. (2016) stated that an average of 3.3 sessions of postinsertion adjustment had needed after 1-year follow-up.

Kattadiyil et al. (2015) stated that 3 of 15 participants preferred conventional full prosthetics than CAD/CAM fabricated CDs. It was reported that in the study of Bidra et al. (2016) 6 of the 17 participants and in the study of Saporano et al. (2016) 4 of the 19 participants had dissatisfaction with CAD/CAM fabricated CD treatment. Kattadiyil et al. (2015) reported that the lower jaw prosthesis of one of the 15 participants was renewed because anterior open bite was observed. Saponaro et al. (2016) stated that the replacement of dentures was necessary for 5 of the 48 participants, as 2 of them had excessive OVD.

\section{Conclusion}

The use of CAD/CAM technology to fabricate complete dentures has positive benefits for both the patient and practitioner. Because the required clinical records can be obtained in one appointment and the dentures completed for a second appointment, there is less clinical time involved in the treatment. Therefore, it should be possible to reduce the cost of care for patients while still providing quality dentures using state, of-the-art dental materials. Elimination of the polymerization shrinkage inherent in conventionally processed completed dentures enhances the fit of the dentures base. Additionally, having a repository of digital data allows for rapid fabrication of spare or replacement dentures.

As we switch to the world of CAD/CAM dentures, competency in making acceptable impressions, determining the appropriate OVD, capturing accurate records, applying esthetic principles and intervention for behavioral modification when required will continue to play a predominant role, even as the applications for this new technology continue to expand.

\section{References}

1. Abduo, J., 2013. Occlusal schemes for complete dentures: A systematic review. Int. J. Prosthodont. 26, 26-33.

2. Al-Helal, A., Al-Rumaih, H.S., Kattadiyil, M.T., Baba, N.Z., Goodacre, C.J., 2016. Comparison of retention between maxillary milled and conventional denture bases: A clinical study. J. Prosthet. Dent. 117, 233-238.

3. Al-Rumaih, H.S., 2016. The effect of denture adhesive on the retention of digital and conventional denture bases: A Cclinical study [thesis]. Loma Linda, CA: Loma Linda University

4. Alghazzawi, T.F., 2016. Advancements in CAD/CAM technology: Options for practical implementation. J. Prosthodont. Res. 60, 7284.

5. Baba, N.Z., 2016. Materials and processes for CAD/CAM complete denture fabrication. Curr. Oral Health Rep. 3, 203-208.

6. Bidra, A.S., Farrell, K., Burnham, D., Dhingra, A., Taylor, T.D., Kuo, C., 2016. Prospective cohort pilot study of 2-visit CAD/CAM monolithic complete dentures and implant-retained overdentures: Clinical and patient-centered outcomes. J. Prosthet. Dent. 115, 578586.

7. Bidra, A.S., Taylor, T.D., Agar, J.R., 2013. Computer-aided technology for fabricating complete dentures: Systematic review of historical background, current status, and future perspectives. J. Prosthet. Dent. 109, 361-366.

8. Bilgin, M.S., Erdem, A., Aglarci, O.S., Dilber, E., 2015. Fabricating complete dentures with $\mathrm{CAD} / \mathrm{CAM}$ and RP technologies. J. Prosthodont. 24, 576-579.

9. Busch, M., Kordass, B., 2006. Concept and development of a computerized positioning of prosthetic teeth for complete dentures. Int. J. Comput. Dent. 9, 113-120.

10. Dankwort, C.W., Weidlich, R., Guenther, B., Blaurock, J.E., 2004. Engineers' CAx education-it's not only CAD. Computer-Aided Design. 36, 1439-1450.

11. Fernandez, M.A., Nimmo, A., Behar-Horenstein, L.S., 2016. Digital denture fabrication in pre- and postdoctoral education: A 
survey of U.S. dental schools. J. Prosthodont. 25, 83-90.

12. Goodacre, B.J., Goodacre, C.J., Baba, N.Z., Kattadiyil, M.T., 2016. Comparison of complete denture base adaptation between $\mathrm{CAD} / \mathrm{CAM}$ and conventional fabrication techniques. J. Prosthet. Dent. 116, 249-256.

13. Goodacre, B.J., Goodacre, C.J., Baba, N.Z., Kattadiyil, M.T., 2018. Comparison of denture tooth movement between CAD-CAm and conventional fabrication techniques. J. Prosthet. Dent. 119, 108115 .

14. Goodacre, C.J., Garbacea, A., Naylor, W.P., Daher, T., Marchack, C.B., Lowry, J., 2012. CAD/CAM fabricated complete dentures: Concepts and clinical methods of obtaining required morphological data. J. Prosthet. Dent. 107, 34-46.

15. Infante, L., Yilmaz, B., McGlumphy, E., Finger, I., 2014. Fabricating complete dentures with CAD/CAM technology. J. Prosthet. Dent. 111, 351-355.

16. Inokoshi, M., Kanazawa, M., Minakuchi, S., 2012. Evaluation of a complete denture trial method applying rapid prototyping. Dent. Mater. J. 31, 40-46.

17. Kanazawa, M., Inokoshi, M., Minakuchi, S., Ohbayashi, N., 2011. Trial of a CAD/CAM system for fabricating complete dentures. Dent. Mater J. 30, 93-96.

18. Kattadiyil, M.T., Goodacre, C.J., Baba, N.Z., 2013. CAD/CAM complete dentures: A review of two commercial fabrication systems. J. Calif. Dent. Assoc. 41, 407-416.

19. Kattadiyil, M.T., Jekki, R., Goodacre, C.J, Baba, N.Z., 2015. Comparison of treatment outcomes in digital and conventional complete removable dental prosthesis fabrications in a predoctoral setting. J. Prosthet. Dent. 114, 818-825.

20. Kawahata, N., Ono, H., Nishi, Y., Hamano, T., Nagaoka, E., 1997. Trial of duplication procedure for complete dentures by CAD/CAM. J. Oral Rehabil. 24, 540-548.

21. Maeda, Y., Minoura, M., Tsutsumi, S., Okada, M., Nokubi, T., 1994. A CAD/CAM system for removable denture. Part I:
Fabrication of complete dentures. Int. J. Prosthodont. 7, 17-21.

22. Muller, F., 2014. Interventions for edentate elders-what is the evidence? Gerodontology. 31 Suppl 1, 44-51.

23. Saponaro, P.C., Yilmaz, B., Heshmati, R.H., McGlumphy, E.A., 2016. Clinical performance of CAD/CAM-fabricated complete dentures: a cross-sectional study. J. Prosthet. Dent. 116, 431-435.

24. Schwindling, F.S., Stober, T., 2016. A comparison of two digital techniques for the fabrication of complete removable dental prostheses: A pilot clinical study. J. Prosthet. Dent.116, 756-763.

25. Srinivasan, M., Cantin, Y., Mehl, A., Gjendegal, H., Müller, F., Schimmel, M., 2017. CAD/CAm milled removable complete denture: An in vitro evaluation of trueness. Clin. Oral Investig. 21, 2007-2019.

26. Steinmassl, P.A., Wiedemair, V., Huck, C., Klaunzer, F., Steinmassl, O., Grunert, I., Dumfahrt, H., 2017. Do CAD/CAM dentures really release less monomer than conventional dentures? Clin. Oral Investig. 21, 1697-1705.

27. Sun, Y., Lü, P., Wang, Y., 2009. Study on CAD\&RP for removable complete denture. Comput. Methods Programs Biomed. 93, 266272.

28. Viola, A.P., Takamiya, A.S., Monteiro, D.B., Barbosa, D.B., 2013. Oral health quality of life and satisfaction before and after treatment with complete dentures in a dental school in Brazil. J. Prosthodont Res. 57, 36-41.

29. Wimmer, T., Gallus, K., Eichberger, M., Stawarczyk, B., 2016. Complete denture fabrication supported by CAD/CAM. J. Prosthet. Dent. 115, 541-546.

30. www.cadazz.com, 2020. CAD software history [Online]. Available at: https:/www.cadazz.com/cad-software-history.htm (Accessed: 21 April 2020).

31. Zhang, Y.D., Jiang, J.G., Liang, T., Hu, W.P., 2011. Kinematics modeling and experimentation of the multi-manipulator tootharrangement robot for full denture manufacturing. J. Med. Syst. 35, 1421-1429. 